\title{
MODELLING OF UNCERTAINTY AND BI-VARIABLE MAPS
}

\author{
Ol'ga Nánásiová ${ }^{*}$ _ Jarosław Pykacz ${ }^{* *}$
}

\begin{abstract}
The paper gives an overview and compares various bi-varilable maps from orthomodular lattices into unit interval. It focuses mainly on such bi-variable maps that may be used for constructing joint probability distributions for random variables which are not defined on the same Boolean algebra.
\end{abstract}

K e y w or d s: orthomodular lattice, probability measure, copula, triangular norm, s-map

\section{INTRODUCTION}

Studies of alternative models for uncertainty (random events) are usefull. We can find many papers on these topics (eg $[15,24,31])$. This paper is devoted to modeling of bi-variate functions for non-compatible or for unsharp events.

In classical (Kolmogorovian) probability theory one works with a single probability space $(\Omega, \mathcal{A}, P)$, where $\Omega$ is a set of elementary events, $\mathcal{A}$ is a Boolean $\sigma$-algebra of subsets of $\Omega$ (random events), and $P$ is a probability measure defined on $\mathcal{A}$. If $X$ and $Y$ are two random variables defined on $(\Omega, \mathcal{A}, P)$ then it is tacitly assumed that $X$ and $Y$ can be measured simultaneously, so their joint distribution function

$$
F_{X, Y}(t, s)=P\left(X^{-1}(-\infty, t) \cap Y^{-1}(-\infty, s)\right)
$$

is well-defined both from theoretical and 'experimental' point of view. Moreover, the famous Sklar's theorem (see, $e g,[23])$ says that even if one knows only marginal distribution functions $F_{X}(t)=P\left(X^{-1}(-\infty, t)\right.$ and $F_{Y}(s)=$ $Y^{-1}(-\infty, s)$ then the joint distribution function $F_{X, Y}$ can be reconstructed.

However, there are situations in which one deals with two or more random variables defined on different probability spaces and, nevertheless, wants to construct a kind of a "joint probability distribution" of them. In such a situation one can build a horizontal sum of Boolean algebras that represent families of random events in these different probability spaces and treat this horizontal sum of Boolean algebras as a single structure that represent random events. However, a horizontal sum of Boolean algebras is not a Boolean algebra but an orthomodular lattice. This fact is a motivation for studying probability measures and random variables on orthomodular lattices. The other motivation comes from quantum physics where orthomodular lattices of closed subspaces of Hilbert spaces represent properties of quantum objects and the existence of random variables (here usually called "observables") that cannot be measured simultaneously is a well-established fact (see, eg, [4,27] and references listed therein).

Investigations presented in this paper have their origin in studies of Nánásiová and her collaborators (see $[18,19,20]$ and, first of all, [1]). We address here the problem of the existence of some useful bi-variable maps introduced in these papers like s-maps and QL-copulas. We also define in this paper bi-variable maps that are generated by classical triangular norms, and study relations between these various maps.

For relevant facts concerning copulas we refer to [23]. Triangular norms were studied in numerous books and papers, eg, in $[7,14,30]$.

\section{BASIC NOTIONS}

We begin with the definition and basic properties of orthomodular lattices $[4,13,27]$.

Definition 1. Let $L$ be a lattice with $I$ (the greatest element), $O$ (the smallest element) and partial ordering $\leq$, endowed with a unary operation $\perp: a \mapsto a^{\perp}$ such that the following conditions are satisfied:

(i) $a^{\perp \perp}:=\left(a^{\perp}\right)^{\perp}=a$;

(ii) $a \leq b$ implies $b^{\perp} \leq a^{\perp}$;

(iii) $a \vee a^{\perp}=1$;

(iv) $a \leq b$ implies $b=a \vee\left(a^{\perp} \wedge b\right)$.

The system $\mathcal{L}=(L, O, I, \leq, \vee, \wedge, \perp)$ is said to be an orthomodular lattice (briefly OML).

Condition (iv) is called the orthomodular law. If it is replaced by the stronger distributivity law: $(a \vee b) \wedge c=$ $(a \wedge c) \vee(b \wedge c)$, then an OML becomes a Boolean algebra. If an OML $\mathcal{L}$ is closed under countable lattice operations, then $\mathcal{L}$ is called a $\sigma$-orthomodular lattice $(\sigma$-OML).

\footnotetext{
* Institute of Computer Science and Mathematics, Faculty of Electrical Engineering and Information Technology, Slovak University of Technology in Bratislava, Ilkovičova 3, 81219 Bratislava 1, Slovakia, nanasiova@math.sk

** Institute of Mathematics, University of Gdańsk, Wita Stwosza 57, 80-952 Gdańsk, Poland, pykacz@mat.ug.edu.pl
} 
Let $a, b \in L$. We say that $a, b$ are orthogonal $(a \perp b)$, if $a \leq b^{\perp}$ and we say that $a, b$ are compatible $(a \leftrightarrow b)$ if $a=(a \wedge b) \vee\left(a \wedge b^{\perp}\right), b=(a \wedge b) \vee\left(a^{\perp} \wedge b\right)$. Let $L^{\prime}$ be a subset of $L$ containing $O$ and $I$. If for each $a, b \in L^{\prime} \quad a \leftrightarrow b$, then $\mathcal{L}^{\prime}$ is a Boolean subalgebra of an OML $\mathcal{L}$, and conversely: every two elements of a Boolean subalgebra of $\mathcal{L}$ are compatible.

Definition 2. Let $\mathcal{L}_{i}=\left(L_{i}, O_{i}, I_{i}, \leq_{i}, \vee_{i}, \wedge_{i}, \perp_{i}\right)$ be a family of orthomodular lattices. The set-theoretic union of all $L_{i}$ in which all smallest elements $O_{i}$ and all greatest elements $I_{i}$ are, respectively, identified, and partial order, lattice operations, and orthocomplementation are inherited from original OMLs $\mathcal{L}_{i}$, is called a horizontal sum of the family of OMLs $\left\{\mathcal{L}_{i}\right\}_{i}$.

It is easy to check that a horizontal sum of OMLs is an OML as well. Therefore, a horizontal sum of Boolean algebras is an OML, although, except of the trivial cases, it is not a Boolean algebra. We show it on the following example.

EXAMPLE 1. Let $\left\{O_{1}, a, a^{\perp}, I_{1}\right\}$ and $\left\{O_{2}, b, b^{\perp}, I_{2}\right\}$ be two different 4 -element Boolean algebras $2^{2}$. Their horizontal sum is a 6 -element orthomodular lattice usually denoted MO2:
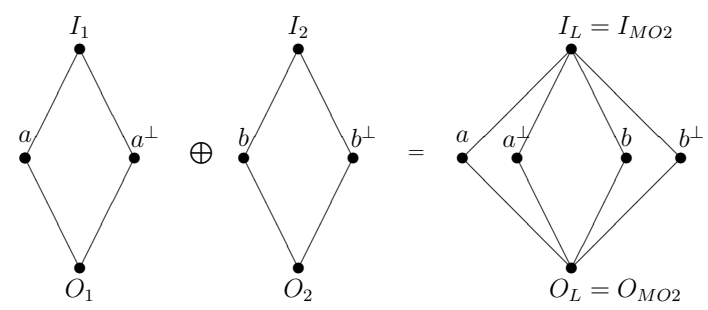

One easily notes that $\left(a \vee a^{\perp}\right) \wedge b=I_{M O 2} \wedge b$ while $(a \wedge b) \vee\left(a^{\perp} \wedge b\right)=O_{M O 2} \vee O_{M O 2}=O_{M O 2}$ so MO2 is not a Boolean algebra.

EXAMPLE 2. Let $\mathcal{B}_{a}=\left\{I_{L}, O_{L}, a_{1}, a_{2}, c, a_{1}^{\perp}, a_{2}^{\perp}, c^{\perp}\right\}$ and $\mathcal{B}_{b}=\left\{I_{L}, O_{L}, b_{1}, b_{2}, c, b_{1}^{\perp}, b_{2}^{\perp}, c^{\perp}\right\}$. It is easy to see that they are Boolean algebras. However, $\mathcal{B}_{a} \cup \mathcal{B}_{b}$ is not a Boolean algebra, but it is an orthomodular lattice.

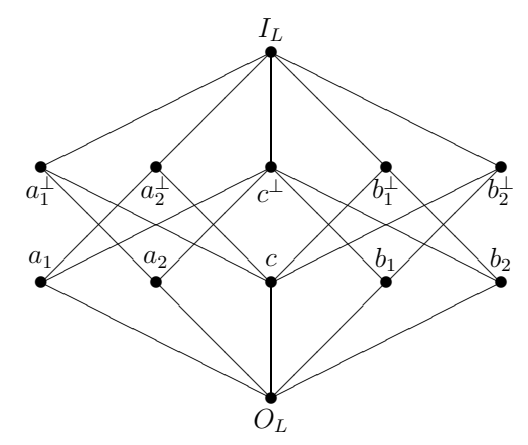

It is possible to see the following facts: $a_{1} \perp a_{2}$ because $a_{1} \leq a_{2}^{\perp}, a_{1} \leftrightarrow a_{2}$ because $\left(a_{1} \wedge a_{2}\right) \vee\left(a_{1} \wedge a_{2}^{\perp}\right)=$ $O_{L} \vee a_{1}=a_{1}$ and since

$$
\left(a_{1} \wedge b_{1}\right) \vee\left(a_{1} \wedge b_{1}^{\perp}\right)=O_{L}<a_{1}
$$

we see $a_{1}$ and $b_{1}$ are not compatible.
Definition 3. Let $\mathcal{L}$ be an $\sigma$-orthomodular lattice. A map $m: L \rightarrow[0,1]$ such that

(i) $m(I)=1$,

(ii) $m\left(\vee_{i=1}^{\infty} a_{i}\right)=\sum_{i=1}^{\infty} m\left(a_{i}\right)$, if $a_{i} \perp a_{j}$ for all $i \neq j$, $a_{i} \in L$

is called a probability measure on $\mathcal{L}$.

Let us note that so defined map on an OML $\mathcal{L}$ is also often called a state on $\mathcal{L}$. If for every $a \in L, m(a) \in$ $\{0,1\}$, then $m$ is called a two-valued probability measure.

Definition 4. A set of probability measures $M$ on an OML $\mathcal{L}$ is called unital if for every $a \in L, a \neq O$ there exists $m \in M$ such that $m(a)=1$, and it is called full or ordering if the following implication holds:

$$
m(a) \leq m(b) \quad \forall m \in M \quad \Rightarrow \quad a \leq b .
$$

It is known, that there exist orthomodular lattices without any probability measure $[12,21]$ and also such that have only one probability measure [21]. If there are at least two different probability measures defined on an OML $\mathcal{L}$, then the set of probability measures defined on $\mathcal{L}$ is obviously uncountable since any convex combination of probability measures is a probability measure as well.

The reader is warned that in [1] an OML with at least one probability measure is called a quantum logic. However, various authors use this name to denote different objects, such as just orthomodular lattices (without mentioning probability measures at all) [32], or orthomodular posets with ordering sets of probability measures [25]. Therefore, we decided not to use this name in the present paper.

\section{S-MAPS}

Functions for simultaneous measurements (abbr. $s$ maps) were introduced in [18] in order to construct joint distribution also for random variables that cannot be measured simultaneously. The definition of an s-map is as follows:

Definition 5. Let $\mathcal{L}$ be an OML. The map $p: L \times L \rightarrow$ $[0,1]$ is called an $s$-map if the following conditions hold:

(S1) $p(I, I)=1$,

(S2) if $a \perp b$, then $p(a, b)=0$,

(S3) if $a \perp b$, then for any $c \in L$

$$
\begin{aligned}
& p(a \vee b, c)=p(a, c)+p(b, c), \\
& p(c, a \vee b)=p(c, a)+p(c, b) .
\end{aligned}
$$

An $s$-map is called commutative iff for any $a, b \in \mathcal{L}$ $p(a, b)=p(b, a)$.

Numerous properties of $s$-maps were studied (see, $e g$, $[1,18-20,28])$. Here we list two of these properties that will be used in the sequel. 
Proposition 1. Let $\mathcal{L}$ be an $O M L$ and let $p$ be an $s$ map on $\mathcal{L}$. Then the following statements are true:

(1) A map $m: L \rightarrow[0,1]$, such that $m(a)=p(a, a)$ for any $a \in L$, is a probability measure on $\mathcal{L}$.

(2) If $a \leftrightarrow b$, then $p(a, b)=p(a \wedge b, a \wedge b)=m(a \wedge b)=$ $p(b, a)$.

Application of $s$-maps, in particular their utilization for constructing joint probability distributions of random variables that are not defined on the same probability space, were studied in numerous papers mentioned above. In the present paper we study the problem of the very existence of $s$-maps, since this problem was a little bit neglected in the previous papers.

In the papers mentioned above numerous examples of $s$-maps defined on various OMLs were given. On the other hand the fact that any $s$-map $p$ generates a probability measure by the formula $m(a)=p(a, a)$ implies that orthomodular lattices with no probability measures also do not admit any $s$-map to be defined on them. The impossibility of defining an $s$-map on some OMLs that have only one probability measure follows from the following lemma.

Lemma 1. Let $\mathcal{L}$ be an $O M L$ and let $p$ be an $s$-map defined on $\mathcal{L}$. If $p(b, b) \neq 0$ then $m_{b}(\cdot): L \rightarrow[0,1]$ defined by $m_{b}(a)=\frac{p(a, b)}{p(b, b)}$ is a probability measure on $\mathcal{L}$ and if $p(b, b) \neq 1$, then the probability measure $m_{b}$ is different from the probability measure $m(\cdot)=p(\cdot, \cdot)$.

Proof. Since $I \leftrightarrow b$, we have by properties of $s$ maps

$$
m_{b}(I)=\frac{p(I \wedge b, I \wedge b)}{p(b, b)}=\frac{p(b, b)}{p(b, b)}=1 .
$$

The fact that $a \perp c \Rightarrow m_{b}(a \vee c)=m_{b}(a)+m_{b}(c)$ follows immediately from the definition of an $s$-map. Finally, we have $m_{b}(b)=\frac{p(b, b)}{p(b, b)}=1$, so it differs from $m(b)=p(b, b)$ if $p(b, b) \neq 1$.

Corollary 1. If $\mathcal{L}$ is an $O M L$ with only one probability measure that is not a two-valued measure, then no $s$-map can be defined on $\mathcal{L}$.

On the other hand every two-valued probability measure defined on an OML $\mathcal{L}$ generates a two-valued and commutative $s$-map on $\mathcal{L}$. Before proving the existence of such an $s$-map we have to remind the notion of the support of a probability measure on an OML (see, eg, $[4])$.

DeFinition 6. Let $m$ be a probability measure on an OML $\mathcal{L}$. We say that $s(m) \in L$ is the support of $m$ if $m(a)=0 \Leftrightarrow a \perp s(m), i e$,

$$
\{a \in L: m(a)=0\}=\{a \in L: a \perp s(m)\} .
$$

It is easy to check that

$$
\{a \in L: m(a)=1\}=\{a \in L: s(m) \leq a\},
$$

so the support of $m$, if exists, is unique.

$\mathrm{R}$ e $\mathrm{m}$ a r k 1. Since in Boolean algebras of sets orthogonality of elements is equivalent to their disjointness, it easy to see that the notion of the support of a probability measure defined on an OML is a straightforward generalization of the notion of the support of a probability measure in a classical probability space $(\Omega, \mathcal{A}, P)$, where $s(P)=\cap\{A \in \mathcal{A}: P(A)=1\}$. However, the support of a classical probability measure always exists, while, for example, a probability measure defined on an atomic OML that takes value 1 on two distinct atoms, has no support.

Proposition 2. Let $\mathcal{L}$ be an $O M L$ and let $M$ be a set of probability measures on $\mathcal{L}$ such that each $a \in L, a \neq O$ is the support of exactly one $m_{a} \in M$. Moreover, let $M$ be such that $\forall a, b, c \in L$ such that $a, b \neq O$ the following implication holds

$$
a \perp b \Rightarrow m_{a \vee b}(c)=m_{a}(c) m_{a \vee b}(a)+m_{b}(c) m_{a \vee b}(b) .
$$

Then the function $p: L^{2} \rightarrow[0,1]$ defined as

$$
p(a, b)= \begin{cases}m_{I}(a) m_{a}(b) & \text { if } a \neq O, \\ 0 & \text { if } a=O\end{cases}
$$

is an $s$-map on $\mathcal{L}$.

Proof. The condition (S1) of the definition of an $s$-map is obvious:

$$
p(I, I)=m_{I}(I) m_{I}(I)=1 .
$$

The condition (S2) follows from the definition of the support: If $a \perp b$ and $a \neq O$, then

$$
p(a, b)=m_{I}(a) m_{a}(b)=m_{I}(a) \cdot 0=0 .
$$

To prove the first equality of (S3) we utilize the assumption (1). Since $(a \vee b) \vee(a \vee b)^{\perp}=I$, we get from (1)

$$
\begin{aligned}
m_{I}(a)=m_{(a \vee b) \vee(a \vee b)^{\perp}}(a)= & m_{a \vee b}(a) m_{I}(a \vee b) \\
& +m_{(a \vee b)^{\perp}}(a) m_{I}\left((a \vee b)^{\perp}\right) .
\end{aligned}
$$

But $a \leq a \vee b=\left((a \vee b)^{\perp}\right)^{\perp}$, so $a \perp(a \vee b)^{\perp}$ and from the definition of the support we get $m_{(a \vee b)^{\perp}}(a)=0$, which means that

$$
m_{I}(a)=m_{a \vee b}(a) m_{I}(a \vee b)
$$

Analogously we get

$$
m_{I}(b)=m_{a \vee b}(b) m_{I}(a \vee b),
$$


and finally

$$
\begin{aligned}
p & (a \vee b, c)=m_{I}(a \vee b) m_{a \vee b}(c) \\
& =m_{I}(a \vee b)\left[m_{a}(c) m_{a \vee b}(a)+m_{b}(c) m_{a \vee b}(b)\right] \\
& =m_{I}(a \vee b) m_{a \vee b}(a) m_{a}(c)+m_{I}(a \vee b) m_{a \vee b}(b) m_{b}(c) \\
& =m_{I}(a) m_{a}(c)+m_{I}(b) m_{b}(c) \\
& =p(a, c)+p(b, c) .
\end{aligned}
$$

It is a little bit surprising that the second equality of the condition (S3) is valid regardless of the assumption (1):

If $a \perp b$, then for any $c \neq O$

$$
\begin{aligned}
p(c, a \vee b) & =m_{I}(c) m_{c}(a \vee b)=m_{I}(c)\left[m_{c}(a)+m_{c}(b)\right] \\
= & m_{I}(c) m_{c}(a)+m_{I}(c) m_{c}(b)=p(c, a)+p(c, b) .
\end{aligned}
$$

R e m ark 2 . Since $m(s(m))=1$, the assumption that each $a \in L, a \neq O$ is the support of a measure $m_{a} \in M$ obviously means that the set of probability measures $M$ is unital.

R e m a r k 3 . Let $(\Omega, \mathcal{A}, P)$ be a classical probability space and let a probability measure $P$ be such that $\forall A \in$ $\mathcal{A}, P(A) \neq 0$ iff $A \neq \emptyset$. Then the set $M$ of conditional probabilities

$$
M=\left\{P_{A}: P_{A}(B)=P(B \mid A)=\frac{P(A \cap B)}{P(A)}\right\}
$$

fulfills assumptions of the previous proposition: Every $A \neq \emptyset$ is the support of exactly one probability measure $P_{A} \in M$, and if $A \cap B=\emptyset$, then for any $C \in \mathcal{A}$

$$
\begin{aligned}
& P_{A \cup B}(C)=P(C \mid A \cup B)=\frac{P(C \cap(A \cup B))}{P(A \cup B)} \\
& =\frac{P((C \cap A) \cup(C \cap B))}{P(A \cup B)}=\frac{P(C \cap A)}{P(A \cup B)}+\frac{P(C \cap B)}{P(A \cup B)} \\
& =P(C \mid A) P(A \mid A \cup B)+P(C \mid B) P(B \mid A \cup B) \\
& =P_{A}(C) P_{A \cup B}(A)+P_{B}(C) P_{A \cup B}(B)
\end{aligned}
$$

so the condition (1) is fulfilled and the bi-variable map defined on $\mathcal{A}$ by

$$
p(A, B)= \begin{cases}P(A) P(B \mid A) & \text { if } A \neq \emptyset \\ 0 & \text { if } A=\emptyset\end{cases}
$$

is an $s$-map defined on $\mathcal{A}$.

We finish this section with a much expected fact, the proof of which is a straightforward exercise.

Proposition 3. Any convex combination of $s$-maps defined on an $O M L \mathcal{L}$ is an $s$-map defined on an $O M L$ $\mathcal{L}$.

\section{QL-COPULAS}

In classical probability a copula function (see [23]) allows to simulate unknown joint distribution function when only marginal distribution functions are known.
Definition 7. A two-dimensional copula is a function $C:[0,1]^{2} \rightarrow[0,1]$ with the following properties

(C1) $C(0, u)=C(u, 0)=0$ for all $u \in[0,1]$,

(C2) $C(u, 1)=C(1, u)=u$ for all $u \in[0,1]$,

(C3) if $u_{1} \leq u_{2}$ and $v_{1} \leq v_{2}$, then

$$
C\left(u_{1}, v_{1}\right)+C\left(u_{2}, v_{2}\right) \geq C\left(u_{1}, v_{2}\right)+C\left(u_{2}, v_{1}\right) .
$$

The importance of copulas in classical probability follows from Sklar's theorem [23]:

THEOREM 1. Let $F$ be a two-dimensional distribution function with marginal distributions $F_{1}$ and $F_{2}$. Then there exists a two-dimensional copula $C$ such that

$$
F(t, s)=C\left(F_{1}(t), F_{2}(s)\right) \text { for every } t, s \in R .
$$

Conversely, for any distribution functions $F_{1}$ and $F_{2}$ and for every copula $C$, the function $F$ given by (2) is a twodimensional distribution function with marginals $F_{1}$ and $F_{2}$. Furthermore, if $F_{1}$ and $F_{2}$ are continuous, then $C$ is unique.

The notion of a QL-copula was defined in [1] as follows.

Definition. Let $\mathcal{L}$ be an OML. A function $C: L^{2} \rightarrow$ $[0,1]$ that satisfies the conditions

(C1) $C(O, a)=C(a, O)=0$ for each $a \in L$,

(C2) $C(I, \cdot), C(\cdot, I)$ are probability measures on $\mathcal{L}$,

(C3) if $a \leq b$ and $c \leq d$, then $C(b, d)+C(a, c) \geq$ $C(a, d)+C(b, c)$,

is called a $Q L$-copula.

If $p$ is an $s$-map defined on an OML $\mathcal{L}$, then the existence of a QL-copula is guaranteed by the following proposition proved in [1].

Proposition 4. Let $\mathcal{L}$ be an $O M L$ and let $p$ be an $s$ map on it. Then $p$ is a $Q L$-copula.

This proposition shows that the notion of a QL-copula is more general than the notion of an $s$-map. We shall show in the sequel in a specific example that there exist QL-copulas that are not not $s$-maps, $i e$, the set of all $s$ maps defined on an OML $\mathcal{L}$ is usually strictly included in the set of all QL-copulas defined on $\mathcal{L}$. The problem of finding general conditions under which a QL-copula would be an $s$-map is, for the time being, not solved.

Although Proposition 4 assures the existence of a QLcopula on an OML $\mathcal{L}$ when there exists an $s$-map on $\mathcal{L}$, one can construct a lot of QL-copulas on $\mathcal{L}$ in a straightforward way, without the neccesity of using $s$ maps. 
Proposition 5. Let $m_{1}, m_{2}$ be two, not necessarily different, probability measures on an $O M L \mathcal{L}$ and let $C:[0,1]^{2} \rightarrow[0,1]$ be a two-dimensional copula. Then a function $C_{m_{1}, m_{2}}: L^{2} \rightarrow[0,1]$ defined for any $a, b \in L$ by

$$
C_{m_{1}, m_{2}}(a, b)=C\left[m_{1}(a), m_{2}(b)\right]
$$

is a $Q L$-copula defined on $\mathcal{L}$.

The proof follows immediately from definitions of twodimensional copulas and QL-copulas, and from properties of probability measures defined on orthomodular lattices.

$\mathrm{R}$ e mark 3 . It was noticed in [1] that if $m_{1}, m_{2}$ are two probability measures on an OML, then $C(a, b)=$ $m_{1}(a) m_{2}(b)$ is a QL-copula. Actually, this follows from the previous proposition since $C(x, y)=x y$ is a twodimensional copula (see, eg, [23]).

ExAMPLE 3. Let $\mathcal{L}$ be an orthomodular lattice MO2 described in Example 1 and let $m_{1}$ and $m_{2}$ be two probability measures defined by the following table.

\begin{tabular}{ccccc}
$x=$ & $a$ & $a^{\perp}$ & $b$ & $b^{\perp}$ \\
\hline$m_{1}(x)$ & 0.2 & 0.8 & 0.3 & 0.7 \\
$m_{2}(x)$ & 0.4 & 0.6 & 0.5 & 0.5 \\
\hline
\end{tabular}

Since bi-variable maps $T_{0}(x, y)=\min (x, y), T_{1}(x, y)=$ $x y$, and $T_{\infty}(x, y)=\max (x+y-1,0)$ are two-dimensional copulas (see, eg, [23]), they generate QL-copulas on MO2 by

$$
C_{m_{1}, m_{2}}(a, b)=T_{i}\left[m_{1}(a), m_{2}(b)\right] \quad i=0,1, \infty,
$$

neither of these maps is an $s$-map on MO2 since it is easy to check that for the QL-copula generated by $T_{0}$ and $T_{\text {infty }}$ the conditions (S2) and (S3) of Definition 5 are not fulfilled, for the QL-copula generated by $T_{1}$ the condition (S2) is not fulfilled.

Note that it is easy to check that the conditions (S2) and (S3) are not fulfilled both for OL-copulas generated by $T_{0}$ and by $T_{\text {infty }}$ t-norms.

We finish this section with a fact, the proof of which is a simple exercise:

Proposition 6. Any convex combination of $Q L$-copulas is a $Q L$-copula.

\section{BI-VARIABLE MAPS ON OMLS GENERATED BY TRIANGULAR NORMS}

Triangular norms, defined by K. Menger already in $1942[16]$, are another maps from $[0,1]^{2}$ into $[0,1]$ that have been, and still are, intensively studied and utilized in various branches of mathematics, like probabilistic metric spaces $[33,30]$, fuzzy sets $[7,17]$, multivalued logic [29], mathematical statistics [9], capacity theory [10], or game theory $[2,3,5,6]$, to mention a few.
Definition 9. A triangular norm (abbr. t-norm) is a function $T:[0,1]^{2} \rightarrow[0,1]$ which is commutative, associative, nondecreasing in each argument, and satisfies the boundary condition: $T(x, 1)=x$.

The most important family of t-norms is the family of fundamental t-norms $\left\{T_{s}\right\}_{s \in[0, \infty]}$ obtained by M. D. Frank [10] as continuous solutions of the functional equation $F(x, y)+1-F(1-x, 1-y)=x+y$. This family is

$$
T_{s}(x, y)= \begin{cases}\min (x, y) & \text { if } s=0, \\ x y & \text { if } s=1, \\ \max (x+y-1,0) & \text { if } s=\infty \\ \log _{s}\left[1+\frac{\left(s^{x}-1\right)\left(s^{y}-1\right)}{s-1}\right] & \text { otherwise. }\end{cases}
$$

This family is "continuous" in the sense that $\lim _{s \rightarrow t} T_{s}=$ $T_{t}$, and t-norms $T_{0}, T_{1}$, and $T_{\infty}$ encountered already in the previous section are distingushed also because they generate on fuzzy sets operations known as Zadeh, probabilistic, and Łukasiewicz intersection, respectively (see, eg, $[7,14,17])$.

Triangular norms and two-dimensional copulas are both functions from $[0,1]^{2}$ into $[0,1]$, but neither of these families of functions contains, nor is contained in the other. However, commutative and associative twodimensional copulas are t-norms, and t-norms that satisfy the 1-Lipschitz condition, ie, $|T(w, x)-T(y, z)| \leq$ $|w-y|+|x-z|$ for all $w, x, y, z \in[0,1]$ are twodimensional copulas [22]. In particular t-norms $T_{0}, T_{1}$, and $T_{\infty}$ are two-dimensional copulas.

It occures [23] that t-norms $T_{0}$ and $T_{\infty}$ are, respectively, the greatest and the least of all two-dimensional copulas since for all $x, y \in[0,1]$

$$
\begin{aligned}
& T_{\infty}(x, y)=\max (x+y-1,0) \leq C(x, y) \leq \\
& \min (x, y)=T_{0}(x, y) .
\end{aligned}
$$

Let us note that for a bi-variable function that maps an OML into $[0,1]$ the notion of associativity makes no sense. Therefore, in order to define bi-variable maps on OMLs that would be "functional counterparts" of classic triangular norms, we have to omit associativity. We adopt the following definition.

Definition 10. Let $\mathcal{L}$ be an OML. A function $T: L^{2} \rightarrow$ $[0,1]$ that is commutative, nondecreasing in each argument, and satisfies the boundary condition

$$
T(\cdot, I) \text { is a probability measure on } \mathcal{L},
$$

is called a T-map.

In the previous section we showed that if we have two (not necessarily different) probability measures on an OML, then any two-dimensional copula generates a $\mathrm{QL}-$ copula. The analogous proposition concerning t-norms and T-maps is as follows. 
PROPOSITION 7. Let $m$ be a probability measure on an OML $\mathcal{L}$ and let $T:[0,1]^{2} \rightarrow[0,1]$ be a triangular norm. Then a function $T_{m}: L^{2} \rightarrow[0,1]$ defined for any $a, b \in L$ by

$$
T_{m}(a, b)=T[m(a), m(b)]
$$

is a $T$-map defined on $\mathcal{L}$.

The proof, as in the case of QL-copulas, follows immmediately from definitions of a t-norm, T-map, and properties of probability measures defined on OMLs.

$\mathrm{R}$ e $\mathrm{m}$ ar $\mathrm{k} 4$. Since there is no counterpart of the notion of associativity in the definition of a T-map, it is obvious that any map $T:[0,1]^{2} \rightarrow[0,1]$ that is commutative but not necessarily associative, nondecreasing in both arguments, and is such that $T(x, 1)=x$, generates a T-map on an OML in a way described in the previous proposition.

R e mark 5. A T-map cannot in general be constructed like a QL-copula was constructed in Proposition 5, ie, with the use of two different probability measures, because of the required commutativity. Although by commutativity of any t-norm we have $T\left[m_{1}(a), m_{2}(b)\right]=T\left[m_{2}(b), m_{1}(a)\right]$, this is in general different from $T\left[m_{1}(b), m_{2}(a)\right]$. In a full analogy to the relation between classical two-dimensional copulas and t-norms we have the following lemma.

Lemma 2. Any commutative QL-copula is a T-map.

P r o o f . Commutativity and the boundary condition are obvious. In order to prove non-decreasingness it is enough to put $c=O$ in the condition (C3) of the definition of a QL-copula, which then takes the form

if $a \leq b$ and $O \leq d$, then

$$
C(a, d)+C(b, O) \leq C(b, d)+C(a, O),
$$

and note that by $(\mathrm{C} 1), C(b, O)=C(a, O)=0$.

Since by Proposition 4 any commutative $s$-map is a commutative QL-copula, we obtain the following corollary

Corollary 2. Any commutative s-map on an OML is a T-map.

The proof of the following much expected fact that is a T-map counterpart of Propositions 3 and 6 , is a straightforward exercise.

Proposition 8. Any convex combination of T-maps is a T-map.

Before closing this section let us note that there are well-known generalizations of classical triangular norms and conorms to bounded posets ( $i e$, partially ordered sets with the least and the greatest elements) in the sense of two-argument operations on posets, not in the sense of $b i$ variable functions from a poset into the unit interval ( $\mathrm{eg}$, $[8,34])$. According to this approach a triangular norm $T$ on a bounded poset $\mathcal{L}=\{L, \leq, O, I\}$ is a two-argument operation on $\mathcal{L}$, ie, a map $T: \mathcal{L}^{2} \rightarrow \mathcal{L}$ that is commutative, associative, nondecreasing, and satisfies the boundary condition $T(a, I)=a$, ie, it satisfies all properties of a t-norm defined on $[0,1]$. Since any OML is a bounded poset, this approach is applicable to OMLs as well, but in this paper we are interested in bi-variable maps from OMLs into $[0,1]$, not in operations on OMLs. Nevertheless, the following proposition can be easily proved.

Proposition 9. Let $\mathcal{L}$ be an OML. Then every probability measure $m$ on $\mathcal{L}$ and every triangular norm $T: L^{2} \rightarrow L$ defined on $\mathcal{L}$ generate a $T$-map $T_{m}: L^{2} \rightarrow$ $[0,1]$ by

$$
T_{m}(a, b)=m(T(a, b))
$$

\section{TRANGULAR NORMS AND COPULAS IN FUZZY SET REPRESENTATIONS OF ORTHOMODULAR LATTICES}

In order to simplify the notation we adopt in the present section the following conventions

- The t-norm $T_{\infty}$ will be denoted $\sqcap, i e, \forall x, y \in[0,1]$, $x \sqcap y=\max (x+y-1,0)=T_{\infty}(x, y)$, and its dual tconorm $S_{\infty}(x, y)=1-T_{\infty}(1-x, 1-y)$ will be denoted $\sqcup$, ie, $x \sqcup y=\min (x+y, 1)=S_{\infty}(x, y)$.

- The same symbols will be used to denote operations on fuzzy sets pointwisely defined by this t-norm and t-conorm, and usually called Łukasiewicz intersection and union:

$$
\begin{gathered}
(A \sqcap B)(x)=A(x) \sqcap B(x)=\max [A(x)+B(x)-1,0], \\
(A \sqcup B)(x)=A(x) \sqcup B(x)=\min [A(x)+B(x), 1] .
\end{gathered}
$$

- The standard fuzzy complement of a set $A$ will be denoted $A^{\prime}$, ie, $A^{\prime}(x)=1-A(x)$.

In [25] the following representation theorem was proved:

ThEOREM 2. Every orthomodular poset $\mathcal{L}$ with an ordering set of probability measures $M$ can be isomorphically represented by a family $\Lambda$ of fuzzy subsets of $M$ endowed with the inclusion of fuzzy sets as partial order, the standard fuzzy set complementation as orthocomplementation, and such that

(i) $\emptyset \in \Lambda$,

(ii) $A \in \Lambda \Rightarrow A^{\prime} \in \Lambda$,

(iii) if $\left\{A_{i}\right\} \subset \Lambda$ is at most countable sequence such that $A_{i} \sqcap A_{j}=\emptyset$ for $i \neq j$ (such fuzzy sets were called weakly disjoint by $R$. Giles in [11]), then $\sqcup_{i} A_{i} \in \Lambda$,

(iv) if $A \in \Lambda$ satisfies $A \sqcap A=\emptyset$, then $A=\emptyset$.

The isomorphism is defined by: $a \mapsto A \Leftrightarrow A(m)=m(a)$.

Since any orthomodular lattice is an orthomodular poset in which operations $\wedge$ and $\vee$ are defined for all pairs of elements, Theorem 2 is obviously valid for any orthomodular lattice $\mathcal{L}$ with an ordering set of probability measures $M$. 
R e m a r k . Orthogonality of two elements $a, b \in \mathcal{L}$ defined by $a \perp b \Leftrightarrow a \leq b^{\perp}$ means that for any probability measure $m$ one has $m(a) \leq m\left(b^{\perp}\right)=1-m(b) \Leftrightarrow$ $m(a)+m(b) \leq 1 \Leftrightarrow m(a) \sqcap m(b)=0$. Therefore, orthogonality of two elements of $\mathcal{L}$ is equivalent to weak disjointness of their fuzzy set representations.

Conditions (i) - (iv) of Theorem 2 can be linguistically expressed as follows.

(i) The empty set belongs to $\Lambda$,

(ii) $\Lambda$ is closed with respect to the standard fuzzy set complementastion,

(iii) $\Lambda$ is closed with respect to Eukasiewicz unions of sequences of pairwisely weakly disjoint sets,

(iv) the empty set is the only set in $\Lambda$ that is weakly disjoint with itself.

Theorem 2 distinguishes Lukasiewicz operations from all other t-norm generated operations on fuzzy sets. Indeed, it can be easily checked that for any other t-norm $T_{s}$ that belongs to the family of Frank's fundamental tnorms, and for any $x \in(0,1)$ one has $T_{s}(x, 1-x) \neq 0$ which means that a $T_{s}$-generated intersection of any genuine fuzzy (ie, non-crisp) set with its complement is nonempty, and which makes $T_{s}$ useless for building fuzzy set representations of orthomodular posets or lattices.

Although Łukasiewicz intersection and union are in fuzzy set representation of an OML only partially defined (it was proved in [26] that if they are globally defined, then $\Lambda$ in which all conditions (i) - (iv) are satisfied is necessarily a Boolean algebra), the fact that in the case of such representation one practically deals with numbers from the interval $[0,1]$ allows now to apply to $\Lambda$ pointwisely any "classical" two-dimensional copula or a triangular norm. It is an interesting question what structures can $\Lambda$ generate in this way, of course if one deletes the very restrictive requirement that the empty set is the only set in the structure that is weakly disjoint with itself.

In the case of t-norms the answer is known: the obtained structure is a $t$-tribe, $i e$, a family of fuzzy sets that contains the empty set and is closed with respect to the standard fuzzy set complementation and countable intersections generated by a t-norm. Such families of fuzzy sets are basic structures in fuzzy probability theory and they were studied in numerous papers (see, eg, $[7,17]$ ). The analogous structures based on the notion of a twodimensional copula instead of a t-norm were not studied yet and the question remains open.

\section{SUMMARY}

In the paper we studied various bi-variable functions that map an OML into the unit interval $[0,1]$ and are generalizations of bi-variable maps defined on $[0,1]$ that are extensively used in classical probability. We showed the hierarchy of notions: each $s$-map is a QL-copula and each commutative $s$-map is a commutative QL-copula and a T-map. The ways in which these functions can be constructed from given probability measures on an
OML were shown. These constructions can be useful for constructing joint distributions of random variables that are not simultaneously measurable.

\section{Acknowledgements}

The first author has been supported by the grant VEGA $1 / 0710 / 15$.

The second author gratefully acknowledges a scholarship granted to him by the Slovak Academic Information Agency under the Slovak National Scholarship Programme which allowed him to stay several months at the Slovak Technical University in Bratislava.

\section{REFERENCES}

[1] AL-ADILEE, A. M.-NÁNÁSIOVÁ, O. : Copula and s-Map on a Quantum Logic, Information Sciences 179 (2009), 4199-4207.

[2] AUBIN, J. P.: Mathematical Methods of Games and Economic Theory, North-Holland, Amsterdam, 1979.

[3] AUMAn, R. J.-Shapley, L. S.: Values of Non-Atomic Games, Princeton University Press, Princeton, 1974.

[4] Beltrametti, E. G.-CASsinelli, G.: The Logic of Quantum Mechanics, Addison-Wesley, Reading MA, 1981.

[5] BUTNARIU, D.: Non-Atomic Fuzzy Measures and Games, Fuzzy Sets and Systems 17 No. 1985, 39-52.

[6] BUTNARIU, D.: Values and Cores for Fuzzy Games with Infinitely Many Players, International Journal of Game Theory 16 (1987), 43-68.

[7] BUTnARIU, D.-KLEMENT, E. P.: Triangular Norm-Based Measures and their Markov Kernel Representation, Journal of Mathematical Analysis and Applications 162 (1991), 111-143.

[8] De BAETS, B.-MESIAR, M.: Triangular Norms on Product Lattices, Fuzzy Sets and Systems 104 (1999), 61-75.

[9] DVORETZKY, A.-WALD, A.-WOLFOWITZ, J.: Relations Among Certain Ranges of Vector Measures, Pacific Journal of Mathematics 1 (1951), 59-74.

10] FRANK, M. D.: On the Simultaneous Associativity of $F(x, y)$ and $x+y-F(x, y)$, Aequationes Mathematicae 19 (1979), 194-226.

[11] GILES, R. : Łukasiewicz Logic and Fuzzy Set Theory, International Journal of Man-Machine Studies 67 (1976), 313-327.

12] GREECHIE, R. J.: Orthomodular Lattices Admitting no States, Journal of Combinatorial Theory, Ser. A 10 (1971), 119-132.

[13] KALMBACH, G.: Orthomodular Lattices, Academic Press, London, 1983.

[14] KLEMENT, E. P.-MESIAR, R.-PAP, E. : Triangular Norms, Kluwer, Dordrecht, 2000.

[15] MARTINEK, R.-KELNAR, M.-VANUS, J. : A Robust Approach for Acoustic Noise Suppression in Speech using ANFIS, Journal of Electrical Engineering 66 (2015), 301-310.

[16] MENGER, K. : Statistical Metrics, Proceedings of the National Academy of Sciences of the USA 28 (1942), 353-537.

[17] MESIAR, R. : Fundamental Triangular Norm Based Tribes and Measures, Journal of Mathematical Analysis and Applications 177 (1993), 633-640.

18] NÁNÁSIOVÁ, O.: Map for Simultaneous Measurements for a Quantum Logic, International Journal of Theoretical Physics 42 (2003), 1889-1903.

[19] NÁNÁSIOVÁ, O.-KHRENNIKOV, A.: Representation Theorem for Observables on a Quantum System, International Journal of Theoretical Physics 45 (2006), 469-482. 
[20] NÁNÁSIOVÁ, O.-VALÁŠKOVÁ, L': Maps on a Quantum Logic, Soft Computing 14 (2010), 1047-1052.

[21] NAVARA, M.: Small Quantum Structures with Small State Spaces, International Journal of Theoretical Physics 47 (2008), $36-43$.

[22] NAVARA, M.: Triangular Norms and Conorms, www.scholarpedia.org/article/Triangular_norms_and_conorms .

[23] NELSEN, R. B.: An Introduction to Copulas, Springer, New York, 1999.

[24] PetroviĆ, I.-JOzSA, L.-BAUS, Z. : Use of Fuzzy Logic System for Assessment of Primary Faults, Journal of Electrical Engineering 66 (2015), 257-263.

[25] PYKACZ, J.: Fuzzy Quantum Logics and Infinite-Valued Łukasiewicz Logic, International Journal of Theoretical Physics 33 (1994), 1403-1416.

[26] PYKACZ,.: Łukasiewicz Operations in Fuzzy Set and ManyValued Representations of Quantum Logics, Foundations of Physics 30 (2000), 1503-1524.

[27] PTÁK, P.-PULMANNOVÁ, S. : Orthomodular Structures as Quantum Logics, Kluwer, Dordrecht, 1991.

[28] PYKACZ, J.-VALÁŠKOVÁ, L.-NÁNÁSIOVÁ, O.: BellType Inequalities for Bivariate Maps on Orthomodular Lattices, Foundations of Physics 45 (2014), 900-913.

[29] ROSE, A.-ROSSER, J. B. : Fragments of Many Valued Statement Calculus, Transactions of the American Mathematical Society 87 (1958), 1-53.

[30] SCHWEIZER, B.-SKLAR, A.: Probabilistic Metric Spaces, North-Holland, New York, 1983.

[31] SOCHA, V.-KUTÍLEK, P.—VITEČKOVÁ, S. : The Evaluation of the Practical Adhesion Strenght of Biocompatible Thin
Films by Fuzzy Logic Expert Sysstem and International Standards, Journal of Electrical Engineering 64 (2013), 354-360.

[32] TKADLEC, J. : Subadditivity of States on Quantum Logics, International Journal of Theoretical Physics 34 (1995), 1767-1774.

[33] WALD, A.: On Statistical Generalizations of Metric Spaces, Proceedings of the National Academy of Sciences of the USA 29 (1943), 196-197.

[34] ZHANG, D.: Triangular Norms on Partially Ordered Sets, Fuzzy Sets and Systems 153 (2005), 195-209.

Received 20 February 2016

Ol'ga Nánásiová was born in Modra, Slovakia in 1955. She graduated from the Comenius University in Bratislava in 1979. She obtained PhD (1987) from the Slovak Academy of Sciences. Since 2000 she is an associate professor at Slovak University of Technology. She is a member of the International Quantum Structures Association. She has published more than 50 original papers. Her main research fields include probability theory, algebraic structures and fuzzy set theory.

Jarosław Pykacz was born in Gdańsk, Poland in 1952. He graduated from the University of Gdańsk in 1976, and here he obtained his PhD (1981) and "doctor habilitatus" (2004) degrees. He was one of the founding fathers of the International Quantum Structures Association and a President of this organisation in the years 2008 - 2010. He has published more than 60 papers and a book "Quantum Physics, Fuzzy Sets and Logic". His research fields include mathematical structures encountered in foundations of quantum mechanics, fuzzy set theory, and quantum game theory. 